Cahiers d'études italiennes

Pétrarque et le pétrarquisme

\title{
Canzoniere : le jeu de la construction
}

\section{Claude Perrus}

\section{OpenEdition Journals}

Édition électronique

URL : http://journals.openedition.org/cei/610

DOI : 10.4000/cei.610

ISSN : 2260-779X

Éditeur

UGA Éditions/Université Grenoble Alpes

\section{Édition imprimée}

Date de publication : 15 avril 2006

Pagination : 9-17

ISBN : 978-2-84310-081-9

ISSN : $1770-9571$

Référence électronique

Claude Perrus, «Canzoniere : le jeu de la construction », Cahiers d'études italiennes [En ligne], 4 | 2006, mis en ligne le 15 octobre 2007, consulté le 20 avril 2019. URL : http://journals.openedition.org/ cei/610 ; DOl : 10.4000/cei.610 


\title{
CANZONIERE : LE JEU DE LA CONSTRUCTION
}

\author{
Claude Perrus \\ Université de la Sorbonne Nouvelle-Paris 3
}

Deux mots, tout d'abord, sur le titre de cette intervention. Il renvoie au jeu de construction, ou pour mieux dire au puzzle que représente le Canzoniere, un ensemble composé à partir de pièces éparses (Rerum vulgarium fragmenta), et aussi au jeu de, dans, la construction, au sens où un emboîtement de menuiserie peut "avoir du jeu», soit par défaut soit par la volonté de l'artisan. De fait, le Canzoniere présente certains aspects aléatoires, qui posent des problèmes au lecteur et lui ouvrent par là même des choix de lecture et d'interprétation.

Certes, on peut parler de construction à propos de nombre de recueils : ceux de Leopardi, de Montale, d'Ungaretti et d'autres, avec tous les réaménagements dont témoignent parfois leurs rééditions successives. Mais le Canzoniere, comme nous le savons, est autre chose qu'un recueil, même sous la forme organique et réfléchie qu'il peut prendre chez d'autres poètes. C'est avant tout un livre, qui a connu une gestation de plus de trente ans, parallèle à celle d'autres travaux plus prestigieux (que Pétrarque appelle l' «altra opera») qui s'inscrivent, eux, dans une culture institutionnelle que Pétrarque entend rénover. Il n'est achevé, juste avant la mort du poète en 1374 , qu'après un ultime remaniement de l'ordre des dernières poésies.

Comme il a été rappelé plus haut, ce que la tradition appelle Canzoniere porte en fait le titre neutre, mais pourtant significatif de Rerum vulgarium fragmenta, qui signifie non pas "fragments d'œuvres en vulgaire», ce qui désignerait des œuvres fragmentales, inachevées, mais ensemble de textes distincts (à la différence d'un poème tel que l'Africa), écrits en langue vernaculaire. Le titre précédent, celui d'une copie due à Boccace, était Fragmentorum liber, un livre donc, au même titre que le Libro chiamato Decameron qui rassemble et ordonne les nouvelles isolées de ce même Boccace. 


\section{Claude Perrus}

Or l'œuvre de Pétrarque comprend deux autres livres du même genre (à savoir composites): les Familiares (des lettres revues, corrigées, voire inventées pour constituer un ensemble) et les Epystole, lettres en vers et en latin. L'idée de ces ouvrages prend forme dans les années 50, en même temps que commence à se préciser le projet, déjà attesté, du Canzoniere. Dans les trois cas l'auteur veut construire, à partir de pièces éparses (qu'il nomme en latin nugae), ce que Marco Santagata ${ }^{1}$ appelle un «objet durable", aussi achevé que les recueils épistolaires de Cicéron ou les élégies de Properce - pour ne citer que deux modèles -, et de conférer la même dignité à des poésies en langue vulgaire.

Ces opérations, comme Pétrarque le dit lui-même dans une lettre (de dédicace à Pandolfo Malatesta) ${ }^{2}$, lui permettent aussi de se réapproprier des textes dont bon nombre (en latin ou en vulgaire) avaient déjà circulé, recopiées avec nombre d'erreurs. Cette réappropriation ne se borne pas, bien entendu, à un geste d'autorité; elle met aussi en jeu (et j'y reviendrai plus tard) un facteur créatif qui est la relecture (en l'occurrence la re-autolecture).

Ce travail a donc été très long (depuis les premiers recueils datant des années 1336 et 1342-1343 jusqu'au manuscrit définitif, établi après plusieurs stades de rédaction entre 1366 et 1374). Au cours de cette élaboration Pétrarque ajoute, bien entendu, des textes. Mais il peut s'agir soit de textes anciens récupérés et réinsérés sans modifications, soit de textes revus et corrigés, soit encore de textes nouveaux composés en fonction de l'ensemble auquel ils sont destinés (à telle ou telle étape de son projet). Je ne citerai que trois exemples de ces derniers: la série des sonnets dits « du pressentiment» (le poète pressent la mort de Laure), nº 246 à 254, série composée longtemps après la mort de Laure en 1348, et qui ont une fonction narrative de prolepse, d'annonce de l'événement fatal, le sonnet 364, qui est une récapitulation, et a une fonction d'analepse, et enfin la série des sonnets de l'aura (194-198) presque tous écrits à une date assez tardive.

Même les fameux sonnets d' "anniversaires", sortes de jalons obsessionnels indiquant le nombre d'années écoulées depuis la première rencontre, n’ont pas tous été nécessairement écrits aux dates qu'ils sont censés marquer.

1. Marco Santagata, I frammenti dell'anima. Storia e racconto nel Canzoniere di Petrarca, Bologne, Il Mulino, 1992. Nous empruntons à ce livre fondamental la reconstitution des étapes de la composition du Canzoniere.

2. De larges extraits de cette lettre, tirée du recueil des Seniles (XIII, 11, écrite en 1373) sont cités et analysés par SANTAGATA, I frammenti dell'anima, p. 282-285. 
Pétrarque peut également supprimer des textes en cours de route, voire les supprimer puis les réintégrer: c'est le cas du sonnet 211, "damnatum » dans un premier temps, puis, dit l'auteur, relu et absout plusieurs années plus tard. Enfin et surtout il peut modifier leur ordre: je citais tout à l'heure l'exemple des 31 derniers textes renumérotés in extremis pour des raisons de cohérence à la fois thématique et narrative, c'est-à-dire pour mieux préparer la voie au texte final, la prière à la Vierge, mais il y aurait bien d'autres exemples à relever.

Tout cela implique des transcriptions et retranscriptions à l'infini, jusqu'au bel exemplaire écrit sur parchemin, et par suite des variantes; dont on peut se faire une idée puisque l'on dispose d'un manuscrit de brouillons comprenant environ 70 textes du Canzoniere et des Triumphi ${ }^{3}$.

La composition d'un recueil "organique", chez tous les auteurs qui s'y attaquent, est à elle seule une méta-écriture des poèmes, même si l'auteur ne change rien à ses textes. Pour le Canzoniere, cette méta-écriture s'accompagne donc de production nouvelle ou d'une réécriture forcenée, impliquant non seulement un travail de lima mais aussi de copiste, auquel Pétrarque s'est livré seul à partir de 1367 . Or ce travail manuel n'est jamais un exercice neutre: il sollicite l'esprit.

Toutes ces procédures impliquent l'existence d'un projet. Celui-ci, sous sa forme élémentaire, consisterait à disposer des poésies d'amour dans un ensemble qui fasse sens. Ici le premier modèle qui vient à l'esprit est la Vita Nova de Dante, qui censure, redistribue, voire modifie des textes poétiques en fonction d'une narration: le récit d'un itinéraire mental et moral.

Mais ici les textes sont assortis d'une narration en prose qui expose les cagioni: les causes, ou mieux encore les occasions contingentes dont seraient nées les poésies. Or Pétrarque, lui, assume la discontinuité, il compte sur la succession des poèmes, leurs connexions, ou leurs échos à distance, pour que se dégage une ligne générale, pari hasardeux car cette ligne devient de plus en plus complexe au fur et à mesure que s'enrichit l'ouvrage.

3. On trouve deux éditions récentes de ces brouillons, publiés sous le titre de Codice degli abbozzi, dans Francesco Petrarca, Trionfi, Rime estravaganti, Codice degli abbozzi, a cura di Vinicio Pacca e Laura Paolino, Milan, Mondadori, 1996, et dans Francesco PetrarCA, Il Codice degli abbozzi. Edizione e storia del manoscritto Vaticano latino 3196, a cura di Laura Paolino, Milan, R. Ricciardi, 2000. 
Seconde différence: l'esprit du projet n'est pas le même. Bien entendu, le parcours que Dante reconstruit est loin d'être linéaire, mais enfin on y lit clairement la découverte progressive, à travers une expérience de sublimation de l'amour, de raisons nouvelles d'écrire, d'une vocation poétique reconfirmée. La visée de Pétrarque est tout autre. Il suffit de lire le sonnet I et la chanson finale à la Vierge, textes dont la place et donc la vocation est fixée assez tôt, pour voir qu'à l'origine se dessinait, au moins dans les intentions de l'auteur, un parcours axé sur la contrition (relative aux errements causés par l'amour ainsi qu'à leurs témoignages poétiques). Son modèle, sur un plan idéologique, serait plutôt celui des Confessions de saint Augustin, autobiographie d'un pécheur qui s'est libéré (objectif jamais atteint par Pétrarque) de tous les attachements terrestres. Certes, dans le Canzoniere, le récit se concentre avant tout sur l'expérience de l'amour, ce qui n'est pas le cas des Confessions, mais Augustin fournit à Pétrarque (dans ses Confessions ainsi que dans d'autres œuvres) les instruments d'une auto-analyse dont j'évoquerai tout à l'heure quelques éléments.

Mais une autre question surgit: y a-t-il véritablement un parcours, entre le sonnet 1 et la chanson 366?

Je vais retracer très rapidement les «dynamiques rédactionnelles» que Marco Santagata reconstitue en détail dans son livre I Frammenti dell'anima.

Il semble que les premières ébauches du Canzoniere, avant la mort de Laure en 1348, n'aient été que des recueils de textes célébrant Laure, et où se déploient à l'origine la thématique et la symbolique du laurier (couronne du poète), avec comme vecteur le mythe d'Apollon (dieu de la poésie) et de Daphné, l'objet du désir, qui lui échappe en se transformant en laurier (le sonnet 34, dédié à Apollon, inaugurait le tout premier recueil). Ce grand thème de la frustration érotique, fondement de toute la tradition lyrique, Pétrarque ne l'abandonnera jamais mais il l'enrichira peu à peu de motifs inédits.

Le livre prend consistance avec le manuscrit achevé vers 1358 et dédié à Azzo da Correggio. Il est inauguré par l'actuel sonnet 1 qui énonce donc, dans une optique rétrospective, un jugement apparemment négatif sur l'expérience de l'amour comme sur les textes qui en portent la trace.

Toutefois, si l'on peut bien distinguer des étapes dans cette rédaction, comme par exemple celle de la chanson 70 et des trois "chansons des yeux" qui suivent, textes où l'amant-poète, comme Dante, semble passer à une phase de louange pure, leur contenu est aussitôt contredit par d'autres textes qui reprennent le thème du tourment amoureux. 
Dans cette version apparaissent les textes «in morte», enregistrant la mort de Laure mais aussi celle du cardinal Colonna, ancien protecteur de Pétrarque. Ce thème de la mort de la femme aimée n'est pas nouveau dans la poésie, même avant Dante (qui lui a conféré, il est vrai, une dimension «inouïe»). Mais Pétrarque traite ce sujet de façon inédite: le sujet découvre que cette mort ne change rien, que c'est une forme nouvelle de la frustration de l'objet du désir et que l'image de Laure morte, qu'elle surgisse comme souvenir, fantasme, apparition en rêve, a le même pouvoir que celle de Laure vivante: la passion survit à son objet, les mécanismes mentaux restent les mêmes.

Et pourtant la poésie (à ce stade du Canzoniere) était censée ne pas survivre à cette mort, comme l'indiquait le sonnet 292, sur lequel se terminait cette rédaction: "Or sia qui fine al mio amoroso canto : / secca è la vena de l'usato ingegno, / et la cetera mia rivolta in pianto" (v. 12-14) ${ }^{4}$.

Dès lors le sonnet 1 , loin d'annoncer un parcours, pourrait être relu après coup comme une simple excusatio d'auteur.

Les rédactions suivantes ne font que confirmer cette impression, Les textes s'organisent de plus en plus par séries (c'est-à-dire en succession) ou par blocs thématiques. Comme l'écrit Adelia Noferi ${ }^{5}$, l'auteur réalise moins une confession autobiographique qu'un autoportrait. Un autoportrait, précisons-le, qui suppose une introspection et une réflexion d'ordre philosophique très rigoureuses, et toujours en mouvement, étroitement liées au travail de l'écriture. On voit ainsi se multiplier les textes sur le deuil et, parallèlement, les textes sur le désir d'écrire et l'impossibilité de le faire, alors même que l'artisan dispose désormais, nous confie-t-il, de toute la maîtrise technique nécessaire (voir le sonnet 304, confirmation du 292). Mais la nouveauté réside précisément dans le fait que Pétrarque inscrive, écrive cette difficulté, fournissant ainsi une sorte de "récit de l'écriture» et de portrait de l'écrivant.

La chanson à la Vierge apparaît dans une rédaction achevée en 1373 (mais elle a été écrite bien longtemps auparavant), avec en marge la mention «in fine ponatur». Précédée jusqu'au dernier moment du sonnet Vago augelletto che cantando vai, elle sera préparée, après la renumérotation dont j'ai parlé, par une petite série de textes pénitentiels qui la ren-

4. "Qu'ore soit donc la fin de mon chant amoureux: / desséchée est la veine du génie coutumier, / et ma cithare s'est convertie dans les larmes». Toutes les citations du Canzoniere d'après Francesco Petrarca, Canzoniere, ed. M. Santagata, Milan, Mondadori, 1996; la traduction d'après PÉTRARQue, Chansonnier, introd. et trad. de Pierre Blanc, Paris, Bordas, 1991.

5. Adelia NoferI, «Il Canzoniere del Petrarca: scrittura del desiderio e desiderio della scrittura", Paragone, a. XXV, n. 296, octobre 1974, p.3-24. 


\section{Claude Perrus}

dront, en quelque sorte, plus plausible. Là, il apparaît à l'évidence qu'un autre modèle a "travaillé» le livre: non plus la Vita Nova, mais la Divine Comédie, avec la prière de saint Bernard à la Vierge. Rappelons que cette prière a pour objet d'obtenir, par l'intercession de Marie, que Dante ait accès à la vision directe de Dieu, évoquée ensuite dans les derniers vers du Paradis: elle n'est donc que l'avant-dernière étape du parcours à travers les royaumes d'outre-tombe. La prière de Pétrarque, qu'il énonce bien sûr en son nom, marque, elle, une arrivée. De plus ce texte, plus que sanctionner la liquidation d'une erreur, au terme d'une dialectique chrétienne du salut, ouvre sur autre chose: l'espoir dans la grâce divine qui seule pourra sauver l'âme du poète, conformément à une théologie tout augustinienne (Pétrarque tentera une autre voie, plus directe, avec les Trionfi).

On peut donc dire avec Marco Santagata que le projet d'un récit de conversion, s'il a bien existé, s'est enlisé,

Et pourtant le livre existe. La division en deux sections a pris de la consistance (avec par exemple l'adjonction des sonnets du pressentiment dont j'ai déjà parlé). Elle a acquis aussi du sens, à un niveau structurel, avec le choix du nombre définitif de textes: 366, qui confere au livre, à défaut de plan, une superstructure: une sorte de canevas symbolique constitué par l'année liturgique. Sur ce point je résumerai brièvement l'hypothèse avancée par divers critiques; Pétrarque, vivement frappé par la coïncidence de deux dates: sa rencontre avec Laure le 6 avril 1327, la mort de Laure le 6 avril 1348, aurait projeté sa longue histoire sur une sorte de diagramme. Cette histoire commencerait le vendredi saint 1327 (voir sonnet $\mathrm{n}^{\circ} 3$ ), jour où il quitte le droit chemin. 264 jours plus tard, c'est-àdire le jour de Noël - naissance du Sauveur - débuterait le difficile retour aux valeurs spirituelles (voir la chanson 264), retour qui s'achèverait le dimanche de la Passion.

Cette projection de toute une vie sur une année donne donc au livre une apparente ossature. Mais elle n'en reflète pas les oscillations : la contrition n'en est que l'un des axes possibles, et le livre repropose à tout instant les contradictions profondes du sujet (sujet de l'amour et sujet de l'écriture).

Et c'est en cela, paradoxalement, que réside son unité: le Canzoniere est un travail sur soi et un travail sur la poésie, à l'enseigne d'une double et permanente interrogation, de la recherche constante de ce que le poète Yves Bonnefoy nomme la "vérité de parole». Une vérité qui n'a rien à voir, soit dit en passant, avec l'exactitude historique ou avec la sincérité immédiate du Je qui parle. 
Pour simplifier mon propos, je vais distinguer ce qui est indissociable: le travail sur soi et le travail sur la poésie.

De ce travail sur soi, on peut trouver les racines et les raisons dans le Secretum, ce dialogue imaginaire avec un Augustinus, saint Augustin, qui incarne en quelque sorte la conscience de Franciscus, François Pétrarque. Achevé vers 1353, il apparaît en filigrane, ou plutôt à la manière d'un palimpseste, sous les pages du Canzoniere. Et dans l'une comme dans l'autre œuvre, l'auteur analyse moins des sentiments qu'il ne décrit des situations de l'esprit, des états de conscience marqués le plus souvent par la dualité, le conflit, et la dispersion. Pour être "présent à soi-même» (devoir moral fixé par saint Augustin comme par Augustinus), il faut d'abord connaître sa propre identité, afin de pouvoir rassembler, comme Franciscus se le propose à la fin du dialogue, les «sparsa fragmenta» de son âme.

Ces états de conscience peuvent se résumer en deux images, récurrentes dans les œuvres de Pétrarque: celle de l'abîme (un état d'aliénation passionnelle où le sujet ne se reconnaît plus lui-même, d'où sa disposition, si souvent évoquée, à la métamorphose, voir entre autres la chanson 23) et celle du labyrinthe (où le sujet se perd).

Le matériel de cette analyse de soi, ce sont les images mentales, ce qui met en jeu les deux catégories de la mémoire et du temps, ou mieux: du sentiment existentiel du temps (voir par exemple le sonnet 355, mais il existe de nombreux autres exemples). Pétrarque a trouvé les fondements de cette analyse dans les œuvres de saint Augustin. D'ailleurs l'Augustinus du Secretum livre une partie de sa phénoménologie des images, distinguant les souvenirs réels, les images rêvées, et les fantasmes (Phantasmata»), qui s'organisent (comme le dira Freud) en scénarios imaginaires. La critique a amplement analysé ces scénarios récurrents dans le Canzoniere.

Il importe toutefois de souligner un point: le livre n'est pas une simple transcription "diaristique» de ces situations, de ces états, des phénomènes mémoriaux; il est leur construction même, la quête renouvelée des fragments de durée et des objets de mémoire, de sorte que sa discontinuité n'est pas, comme on l'a trop souvent dit, le signe d'une incapacité à construire mais la loi constitutive de ces textes, quelle que soit la date de leur composition. 


\section{Claude Perrus}

J'en viens au travail sur la poésie.

$\mathrm{Au}$ cours de sa riche analyse, Stefano Agosti ${ }^{6}$ a pu observer que chez Pétrarque le projet narratif s'opposait inévitablement, comme un programme un peu extérieur, au caractère «absolu » de l'expérience poétique, inscrit dans le travail du style, au cœur de chaque texte, et qui est la marque spécifique de Pétrarque.

Je voudrais pour ma part souligner l'«actualité permanente», si l'on peut dire, de ce travail. Pétrarque, en effet, ne se borne pas à retranscrire, réordonner, corriger à froid des textes. Ce sont ses textes qu'il revoit, dans tel ou tel état de ce que Paul Valéry appelait l'«opération intérieure» (il faut croire au témoignage des poètes autant qu'aux analyses des critiques). Cet état, qu'il soit euphorique ou dysphorique, implique toujours la vigilance autocritique de l'artisan, avec sa science des nombres et sa règle à calcul (voir par exemple le cas du sonnet 155, dont le poète avait songé à inverser les quatrains, idée abandonnée ensuite "propter sonum ", à cause du son : cela aurait placé les vers les plus sonores au centre du texte, "ce qui est contre la rhétorique", écrit Pétrarque dans une glose marginale du brouillon).

La relecture par un auteur de ses propres textes, disait encore Valéry, est "un dialogue avec les parties réalisées de soi-même». Elle réactualise, par le jeu de superposition de l'état d'âme actuel et de l'état d'âme de la première rédaction, l'instant capturé par celle-ci. C'est une opération de «riscatto memoriale» (j'emploie ici les termes de Gianfranco Folena ${ }^{7}$ ) qui n'a jamais de terme. Aussi la relecture réactive-t-elle les images, ou les symboles, suggérant de nouvelles connexions entre textes, suscitant l'invention de textes nouveaux, par sédimentations successives.

Qui écrit, au cours de ce travail ? Ce n'est évidemment pas le moi empirique mais le sujet de l'écriture, celui qui s'inscrit dans ce discours "autre", que Pétrarque appelait l'«alieniloquium", à savoir le langage de la poésie, que les hommes ont inventé un jour pour s'adresser aux Dieux (Fam. I, 1, 46). En marge du Triomphe de la Renommée, Pétrarque note ceci: «Scribo non tanquam ego, sed quasi aliud».

Autrement dit, le jeu de la construction doit se mesurer sans cesse à la réactualisation des textes, et des fragments de durée dont ils sont dépositaires. Et si la caractéristique de l'histoire, ici, est d'être bloquée comme

6. Stefano Agosti, Gli occhi le chiome, Milan, Feltrinelli, 1993.

7. Gianfranco FolEnA, «L'orologio del Petrarca» [1979], in IDEM, Textus testis. Lingua e cultura poetica delle origini, Turin, Bollati Borighieri, 2002, p. 266-289, p. 282. Cette étude porte sur la conception psychologique et existentielle du temps chez Pétrarque. 
expérience toujours renouvelée du désir frustré et de l'aliénation dont je parlais, cette expérience est justement, comme l'a fait observer Enrico Fenzi ${ }^{8}$, l'objet même de la recherche et le contenu de l'histoire. Et l'image poétique, redécouverte ou inventée, est une prise de possession, une capture, ou captation, mais toujours provisoire, de la chose et de soi-même.

La construction du Canzoniere offre donc, en fin de compte, tellement de «jeu», qu'en dépit du patient labeur de marqueterie accompli par son auteur, elle autorise une lecture libre, anthologique, au choix de chacun.

Mais pour que cette lecture prenne vie, il importe de projeter sur chaque texte le souvenir, l'ombre, et (pourquoi pas?) le fantasme de cet ensemble, qui seul fait sens, et qui est à l'opposé du pétrarquisme des imitateurs. 